\title{
UNE BELLE GAULOISE... \\ À PROPOS D’UNE FIBULE INSCRITE DE LAON
}

\author{
PAR \\ Michel FEUGÈRE \\ Pierre-Yves LAMBERT
}

Une fibule gallo-romaine a été découverte en surface sur un site antique de Laon, «derrière les ateliers de la Moncelle» (Aisne) (FIG. 1). Il s'agit d'un objet en alliage de cuivre, long de 40,5 mm, appartenant à un type à charnière bien connu dans sa forme générale (type F.28b). Il se rattache en effet à un groupe de fibules représentant une semelle de chaussure, prolongé d'une légère excroissance à l'emplacement du talon. Les fibules de cette forme sont généralement des objets émaillés, sur lesquels on remarque que la couleur est souvent parsemée de ponctuations en verre, évoquant les clous.

Cet exemplaire se distingue de la plupart des autres objets connus dans cette série par la présence d'un décor en relief, apposé (par estampage ou au repoussé) sur une feuille de tôle très fine $(0,25 \mathrm{~mm})$ en alliage cuivreux, ultérieurement fixée par brasure sur la face supérieure de la fibule; il s'agit des objets regroupés par E. Riha, dans sa classification des fibules d'Augst, sous le type 7.24 (Riha 1979, 200). Ce dispositif, très fragile, nécessitait une préparation du support que l'on peut observer ici, dans les lacunes du décor, sous la forme de très fines rayures parallèles, un procédé couramment employé pour préparer une soudure ou un collage, en menuiserie par exemple. Nous reviendrons ultérieurement sur les caractéristiques techniques de ce type de décor. Les deux faces de la fibule ont été étamées, ce qui lui donnait l'aspect d'un objet en argent tout en la protégeant de la corrosion.

L'intérêt de la fibule de Laon vient de la présence, dans ce décor, d'une inscription en relief, enserrée dans un cartouche de ponctuation dont chaque extrémité est marquée d'une feuille ou globule. Ce cartouche ponctué, avec la ligne de ponctuations qui souligne la forme de la semelle sur tout son pourtour, évoque une des variantes possible des clous en fer qui équipent très généralement les chaussures gallo-romaines. Le cloutage, qui couvre toute la surface portante, suit très souvent un contour décoratif, dont on connaît plusieurs variantes. Il était donc naturel que les ponctuations soient agencées selon le même principe.

La fin de l'inscription est bien conservée, à l'exception de la dernière lettre qui est incomplète en bas: VIMPI. Le début est plus dégradé, et seules subsistent les premières hastes, obliques mais dans des orientations différentes, des deux premières lettres; une troisième a entièrement disparu, mais ce qui reste permet de restituer sans grande hésitation AVE, peut-être sous la forme AVII. L'inscription aue uimpi est en 
effet déjà attestée sur plusieurs objets gallo-romains, trois fibules et une fusaïole (RIG II-2, L-122). Cette dernière, un objet en terre cuite provenant de la colonie romaine de Nyon (Col. Iulia Equestris) comporte deux lignes écrites en sens contraire, les lettres du mot aue étant séparées par des doubles traits.

Les fibules portant la même inscription que la nôtre sont des objets de forme différente. Celle de Reims, marquée aue ui(m)pi (CIL XIII, 10 027.155), est aujourd'hui perdue. Celle qui a été retrouvée à Cirencester en 1885, portant l'inscription au(e) uimpi (RIB II-3, 2421.41), est une petite fibule à charnière, d'un type indiscutablement féminin, portant une inscription finement ponctuée, datable de la fin du $\mathrm{I}^{\mathrm{er}}$ ou début $\mathrm{du} \mathrm{II}^{\mathrm{e}}$ s. de notre ère. S. Martin-Kilcher $(1998,148)$ a remarqué que les onze fibules connues de cette forme - son groupe B (Riha 5.11) - portent une inscription ponctuée. Il s'agit donc d'une production homogène, faite pour porter de petits messages, qui sont tous des mots d'amour. Aucun d'entre eux n'est personnalisé, par exemple avec le nom de la personne aimée, ce qui nous oriente déjà vers le sens général de la nouvelle inscription de Laon. Cette fibule entre dans la catégorie des objets portant une formule passe-partout, qui prendra tout son sens pour l'utilisateur, mais qui n'est pas rédigée pour un client en particulier (Feugère 2004). Le corpus des fibules à inscription ponctuée fournit deux autres exemples de salutations commençant comme la nôtre: auii / amica / miia (Châtelet de Gourzon); aue te (Tintigny, Lux.) (MartinKilcher 1998, 154).

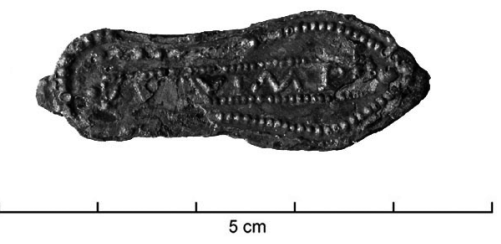

Les fibules en forme de semelle, qui sont datées par leurs contextes des années 60 à 130 environ, sont diffusées dans une vaste région comprise entre le bassin de la Loire, le plateau Suisse et les Flandres, soit l'Est de la Lyonnaise et le Sud de la Gaule Belgique (Feugère 1985, 376, carte fig. 57). Les fibules inscrites du groupe Riha 5.11 se rencontrant exactement dans la même région, la présence d'inscriptions de ce type sur des fibules en forme de semelle n'a rien pour surprendre (Martin-Kilcher 1998, 151, carte fig. 3). E. Riha a publié en 1979 une fibule du Musée historique de Bâle, fabriquée selon la même technique, et portant une inscription incomplète, qui est cependant différente de celle de Laon (Riha 1979, 201, fig. 3.2). Le procédé de fabrication de ces objets doit nous retenir un moment.

Bien que peu étudiée à ce jour, la technique du décor apposé en relief sur une fine feuille ultérieurement collée sur son support, apparaît sur de nombreuses catégories d'objets. Quelques boîtes à sceau, d'époque augustéenne, en apportent apparemment les premiers exemples. Certaines d'entre elles proviennent des niveaux de Kalkriese associés au "désastre de Varus »: on y reconnaît des bustes tournés vers la droite (Schlüter 1992, 373, fig. 15). Plus récemment, une boîte de Lyon, également datée de l'époque augustéenne, a révélé un buste de Mercure, tourné vers la gauche, associé à l'inscription: SCAEVALI (ou VALII) (Desbat 2003). 
Dans le domaine des fibules, on la voit apparaître, à l'époque de Claude, sur des modèles à queue de paon (type F20c), avec des décors qui puisent généralement dans le répertoire des venationes; ces fibules bien représentées dans l'Est de la Gaule ont été largement diffusées dans les régions voisines (Feugère 1985, 294 s., fig. 36 et carte, fig. 37 ; pl. 106 s.). Beaucoup de ces décors fragiles ont disparu et on ne connaît parmi les survivants aucune inscription. Un exemplaire de La Canourgue (Lozère) porte une marque de fabricant estampée au revers, DARIB (ibid., pl. 106, $n^{\circ}$ 1389). Plus rares, des fibules discoïdales de la même époque peuvent porter un décor estampé rapporté, comme c'est le cas dans une tombe récemment mise au jour à Nîmes (Manniez 2005, fig. 2, n 26 : tête de Méduse). Un exemplaire dont le décor a été décrit comme étant en argent, a été découverte à Alésia dans un niveau du milieu du $\mathrm{I}^{\mathrm{er}}$ siècle (Lerat 1979, $\mathrm{n}^{\circ} 311, \mathrm{pl}$. XXXVI). Là encore, il est clair que de nombreux objets aujourd'hui apparemment inornés ont pu comporter un tel décor, entièrement disparu. Toujours dans cette série, une fibule de Cuménal (Alpes-de-Haute-Provence) est ornée d'un médaillon, d'aspect monétiforme, autour de laquelle on lit DEMIOACC / FECIT AV : il s'agit apparemment d'une formule mixte, associant salutation et marque de fabricant (Feugère 1985, pl. 152, n 1914).

Pour les fibules en forme de semelle, enfin, on ne connaît à ce jour que les exemplaires de Laon et de Bâle qui présentent un décor estampé rapporté, mais E. Riha a déjà noté que les fibules à face supérieure lisse, qui n’ont reçu ni émail ni décor gravé, étaient sans doute pourvues d'un tel décor disparu. Un exemplaire d'Augst, qui est dans ce cas, est daté de l'époque de Néron au début des Flaviens (Riha 1979, 200 et $\mathrm{n}^{\circ} 1727$; v. également Riha 1994, 171, n² 2912).

On dispose donc d'indices permettant de situer la technique du décor sur feuille rapportée tout au long du $\mathrm{I}^{\mathrm{er}} \mathrm{s}$. de $n$. ère. Au sein de cette série, les fibules en forme de semelle comptent nécessairement parmi les plus tardifs, ce qui permet de dater la fibule de Laon, comme celle d'Augst, des années 60-90 de n. ère.

\author{
Michel FEUGÈRE \\ UMR 5140 du CNRS \\ 34570 Lattes \\ michel.feugere@wanadoo.fr
}

\title{
AVE VIMPI : deux mots, deux langues
}

vimpi est un adjectif correspondant au gallois gwymp «joli»; ses apparentements indo-européens sont incertains (voir les hypothèses signalées dans RIG-II, fasc. 2, p. 321-322). Pour la désinence, il est probable que nous avons affaire à un féminin, au vocatif singulier. Les formes produites par ce thème dans l'onomastique gallo-romaine (Vimpius, Vimpia, Vimpilla...) n'imposent absolument pas de reconstruire un thème en - $i$-. D'autre part, la séquence VIMPOTA sur un peson de fuseau (L-121) est d'analyse trop douteuse pour imposer de reconstruire un thème en -o-, au masc., en - $\bar{a}$, au 
fém. On retiendra néanmoins cette dernière hypothèse parce qu'elle est conforme au cas le plus fréquent. Donc, posons wimpo-, fém. wimpa.

Dans le gaulois du I ${ }^{\text {er }}$ siècle, la déclinaison des thèmes en - $\bar{a}$ a subi une modification importante résultant d'une simplification générale du système flexionnel: les thèmes en - $\bar{a}$ prennent la flexion des thèmes en -i $i \bar{a}$, c'est-à-dire qu'en dehors du nominatif singulier, tous les cas sont identiques (Lejeune, 1985). Ainsi à l'Hospitalet du Larzac, Paulla et Adiegia ont le même génitif singulier en -ias. Il existe un modèle théorique bien connu pour cette évolution: c'est la flexion des féminins en vieil-irlandais. Dans cette flexion féminine gauloise issue des thèmes en -ia , il apparaît plusieurs désinences qui pourraient dénoncer une identité (structurale) entre thèmes en - $\bar{l}$ - long et thèmes en -iā : l'acc. sg. -im, le vocatif singulier -i, et peut-être un acc. pl. en -is (pannis La Graufesenque) - uniquement des cas directs.

Pour rendre compte de la séquence «Daga vimpi» (L-120) où le premier adjectif a conservé la flexion des thèmes en $-\bar{a}$, nous avons suggéré l'explication suivante (Lambert, 2007): la différence entre daga et vimpi, tous deux au même cas vocatif singulier, provient d'une différence fonctionnelle, le premier étant adjectif, le second étant un adjectif substantivé (= français «la belle»). De même que le français, comme la plupart des langues romanes, utilise l'article pour tirer un substantif d'un adjectif, certaines langues comme l'iranien, le germanique ou le balto-slave ont recours à une forme spéciale de l'adjectif, suffixée par un pronom de thème -io- : c'est la déclinaison faible de l'adjectif allemand, ou l'izafat des langues iraniennes (où il y a suffixation d'une particule -io-). On doit cependant objecter que dans la séquence nata uimpi, plus fréquente (L-112, 118, 121), ou Taurina uimpi (L-113), uimpi accompagne un nom, nom commun (nata «fille») ou nom propre (Taurina), ce qui rend la substantivation inutile; force est donc de constater que la suffixation que nous avons supposée n'est plus liée à une fonction particulière de l'adjectif, mais plutôt à sa place finale dans le syntagme. Il s'agit en quelque sorte d'une extension d'emploi. D'ailleurs, il n'est pas impossible que l'évolution de la flexion des thèmes en $-\bar{a}$ substantifs se soit modelée sur la flexion particulière des adjectifs substantivés, qui n'était pas en $-\bar{a}$, mais en $-i \bar{a}$.

AVE VIMPI présente incontestablement le mélange de langues caractéristique des textes gaulois populaires et/ou tardifs (cf. Meid, 1980). La question d'un sens possible du mot aue en gaulois doit certainement être posée, mais c'est une question qui reste nécessairement théorique étant donné notre faible connaissance de cette langue; les réponses, si elles existent, ne peuvent conduire qu'à un sens secondaire, en contrepoint du sens principal latin. Les formules de salutation gauloises nous sont inconnues; elles sont étroitement codifiées dans les langues celtiques insulaires, (et l'on relève même des archaïsmes, par ex. dans la traduction irlandaise de «Ave Maria »: Is é do bheatha, pour un plus ancien Dé do bhethu « De Dieu, ta vie (?) ») mais aucune ne se rattache à un thème verbal $a w$-. On connaît un thème $a w$ - signifiant offrir (gall. rym-awyr), protéger (irl. con-oi), et un autre thème $a w$ - signifiant souhaiter, désirer (cf. Aventia)... Ainsi l'hypothèse d'un sens secondaire, par le gaulois, se heurte à de nombreuses difficultés et aucune solution convaincante n'en peut assurer la validité.

Comme je l'ai déjà dit RIG II, fasc. 2, p. 319, je crois peu probable que vimpi soit un nom propre, suggestion de Monique Dondin-Payre (Dondin-Payre, 2002). Cette 
démarche obéissait à une vieille tradition des épigraphistes du monde latin, consistant à poser en principe que tout ce qui n'est pas latin dans les inscriptions de Gaule doit être onomastique: on en a un bel exemple dans P. Wuilleumier, ILTG, 529-530, voir aussi RIB II, 3, 1991, 2421.41 pour la fibule de Cirencester. Mais si l'on réfléchit au type de support, (et au cas particulier des petits objets: fibules, pesons), au mode de production des inscriptions (dans tous ces cas portées par les producteurs même de l'objet), et à la fonction du texte inscrit, facilement prévisible, il devient possible de distinguer très nettement entre deux possibilités: la dédicace ou la salutation passe-partout, et la dédicace personnalisée. C'est le mode de production (fibule coulée dans un moule) qui impose la première solution dans le cas de cette inscription AVE VIMPI. Dans le cas d'un mode de production admettant la gravure d'une inscription personnalisée, faite à la demande du client, il suffira de s'en tenir aux statistiques : les pesons comportant Taurina, Maternia sont uniques; mais les pesons comportant les noms nata / gnatha, et vimpi sont multiples: quatre exemples de nata (plus 1 gnatha, 1 genetta), et sept exemples de vimpi. Un huitième exemple de vimpi a été publié, provenant d'Amiens (Binet et Dondin-Payre, 2002). Par conséquent, la multiplicité des occurrences de vimpi sur les pesons, et son occurrence dans un texte de fibule, à quoi l'on ajoutera (argument subsidiaire en faveur de la banalisation du texte) l'identité de légende entre ces fibules et l'un des pesons, tous ces éléments imposent incontestablement de considérer vimpi comme un nom commun (un appellatif).

Il faut donc reconnaître que vimpi est un nom commun, non seulement de provenance gauloise, mais même de flexion gauloise, et c'est précisément ce fait qui « interpelle» le linguiste : l'inscription ne se contente pas d'un «emprunt » au gaulois, mais elle incorpore tel quel un mot gaulois avec sa désinence. On parle souvent de «xénisme», mais l'expression n'est peut-être pas heureuse pour un mot venant de la langue quotidienne. En effet, aue uimpi constitue un mélange de langues qui représente probablement le bilinguisme des couches populaires: un bilinguisme inégal, anarchique, où l'on passe d'une langue à l'autre selon la commodité, ou en fonction de préférences difficiles à préciser. Ces messages mêlés acceptent sans vergogne xénismes et barbarismes. Les exemples abondent, dans les pays celtiques modernes, de ces discours mêlés où l'on passe du gallois à l'anglais, du breton au français dans la même phrase. Les seules conclusions certaines auxquelles nous arrivons, c'est que le mélange de langues est typique d'un message de style familier, ou d'un locuteur peu soucieux d'observer l'unité de langue et de grammaire; on a affaire à un artisan bilingue, dont les clients sont bilingues, mais d'un bilinguisme spontané, populaire, qui admet le mélange de langues dans la même phrase. Il ne s'agit pas du tout du bilinguisme des personnes cultivées, qui, dans chaque langue, observent le purisme et la grammaire. Le retour au gaulois dans la fin du message est donc symptomatique d'un niveau socio-culturel assez simple; il est possible aussi que l'emploi du gaulois révèle qu'une certaine valeur affective s'attache au mot uimpi.

\author{
Pierre-Yves LAMBERT \\ UMR 8546 AOROC (CNRS-ENS) \\ Lambert.pierre-yves@wanadoo.fr
}




\section{BIBLIOGRAPHIE}

Desbat 2003: A. Desbat, «Une boîte à sceau signée d'époque augustéenne», Instrumentum 18, déc. 2003 , p. 40.

Feugère 1985: M. Feugère, «Les fibules en Gaule méridionale de la conquête à la fin du v siècle après J.-C.», Rev. Arch. Narb., suppl.12, Paris, 1985.

Feugère 2004: M. Feugère, «L'instrumentum, support d'écrit », Gallia 61, 2004, p. 53-65.

Lerat 1979: L. Lerat (avec la coll. de F. Blind et E. Rabeisen), Les fibules d'Alésia dans les Musées d'Alise-Sainte-Reine (Bibl. Pro Alésia, VII), Semur-en-Auxois-Dijon, 1979.

Manniez 2005: Y. Manniez, «Essai d'interprétation d'un lot de mobilier d'offrande issu d'une tombe nîmoise du I ${ }^{\text {er }}$ SIÈCLE de n. ère (F)», Instrumentum 21, juin 2005, p. 34-36.

MARTIN-KILCHER 1998: S. Martin-Kilcher, «AB AQUIS VENIO - zu römischen Fibeln mit punzierter Inschrift », in R. Ebersbach, A.R. Furger (Hrsg.), Mille Fiori. Festschr. L. Berger (Forsch. Augst 25), Augst 1998, 147-154.

Rıна 1979: E. Riha, Die römischen Fibeln aus Augst und Kaiseraugst (Forsch. in Augst, 3), Augst 1979.

Rıна 1994: E. Riha, Die römischen Fibeln aus Augst und Kaiseraugst. Vol. 2: Die Neufunde seit 1975 (Forsch. in Augst, Bd. 18), 1994, 206 p., 6 fig., 51 pl.

SchlÜter 1992 : H. Schlüter et al., « Archäologische Zeugnisse zur Varusschlacht? », Germania 70, 1992, p. 307-402.

Binet - Dondin-Payre 2002 : Éric Binet, Monique Dondin-Payre, «La première fusaïole inscrite trouvée à Amiens", Revue du Nord - Archéologie de la Picardie et du Nord de la France, t. 84, 2002, n 348, p. 133-137.

Dondin-Payre 2001: Monique Dondin-Payre, «Fusaïoles et assonance: un problème exemplaire de méthode", in, Dondin-Payre, Monique, Raepsaet-Charlier, Marie-Thérèse, éd., Noms, identités culturelles et romanisation sous le Haut Empire, Bruxelles, Timperman, 2001, p. 318-328, 333-341.

ILTG: Pierre Wuilleumier, Inscriptions latines des Trois Gaules, Paris, Éd. du CNRS, 1963, 19842 (XVII ${ }^{\mathrm{e}}$ supplément à Gallia).

Lambert 2007: Pierre-Yves Lambert, «Emprunts et xénismes dans une épigraphie latine périphérique: les désinences gauloises dans les inscriptions latines gallo-romaines ", in, Fernandez-Vest, M. M. Jocelyne, éd., Combat pour les langues du monde, Fighting for the world's languages, Hommage à Claude Hagège, p. 305-316.

LEJEune 1985: Michel Lejeune, «Notes d'étymologie gauloise, X. La première déclinaison celtique », Études Celtiques XXII, p. 181-194.

MEID 1980: Wolfgang Meid, Gallisch oder Lateinisch? Soziolinguistischen und andere Bemerkungen zu populären gallo-lateinischen Inschriften, Innsbruck, 1980 (Innsbrucker Beiträge zur Sprachwissenschaft, Vorträge und Kleinere Schriften, 24).

RIG II, fasc. 2: Pierre-Yves Lambert, Recueil des Inscriptions Gauloises, vol. II, fasc. 2: Textes gallo-latins sur instrumentum, Paris, CNRS-Éditions (XLV Supplément à Gallia) : p. 317335. 